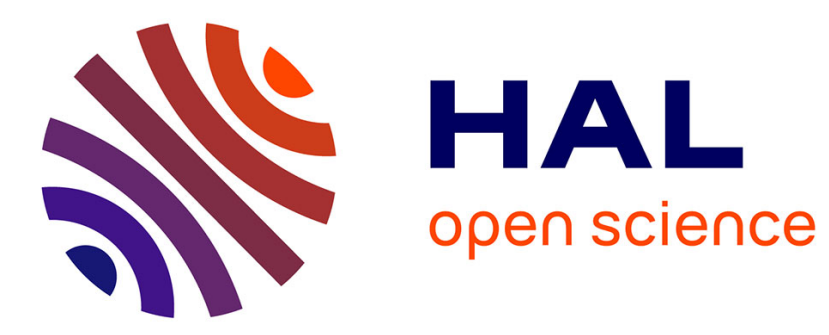

\title{
Harmonic Balance-Based Approach for Quasi-Periodic Motions and Stability Analysis
}

\author{
Mikhail Guskov, Fabrice Thouverez
}

\section{To cite this version:}

Mikhail Guskov, Fabrice Thouverez. Harmonic Balance-Based Approach for Quasi-Periodic Motions and Stability Analysis. Journal of Vibration and Acoustics, 2012, 134 (3), pp.031003-1-031003-11. 10.1115/1.4005823 . hal-00740305

\section{HAL Id: hal-00740305 \\ https://hal.science/hal-00740305}

Submitted on 21 Sep 2015

HAL is a multi-disciplinary open access archive for the deposit and dissemination of scientific research documents, whether they are published or not. The documents may come from teaching and research institutions in France or abroad, or from public or private research centers.
L'archive ouverte pluridisciplinaire HAL, est destinée au dépôt et à la diffusion de documents scientifiques de niveau recherche, publiés ou non, émanant des établissements d'enseignement et de recherche français ou étrangers, des laboratoires publics ou privés. 


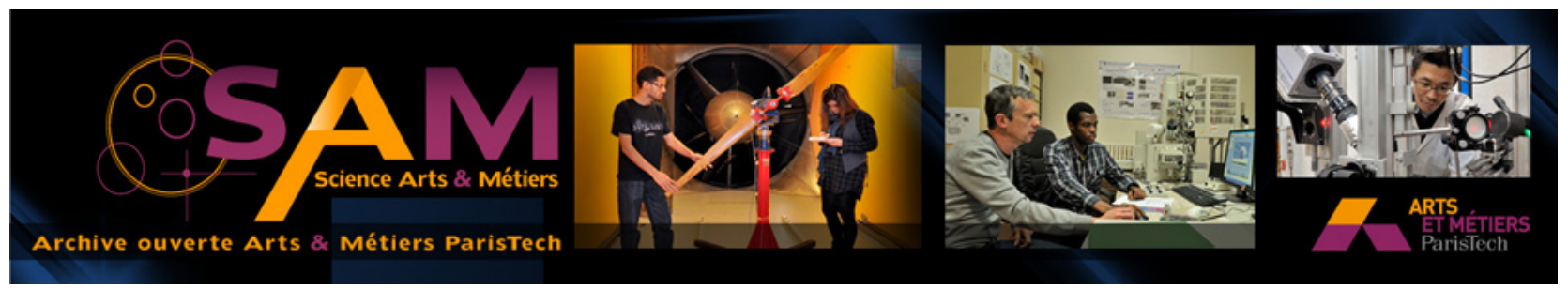

\section{Science Arts \& Métiers (SAM)}

is an open access repository that collects the work of Arts et Métiers ParisTech researchers and makes it freely available over the web where possible.

This is an author-deposited version published in: http://sam.ensam.eu

Handle ID: .http://hdl.handle.net/10985/10080

\section{To cite this version :}

Mikhail GUSKOV, Fabrice THOUVEREZ - Harmonic Balance-Based Approach for Quasi-Periodic Motions and Stability Analysis - Journal of Vibration and Acoustics - Vol. 134, p.031003-1031003-11 - 2012 


\section{Mikhail Guskovour Harmonic Balance-Based \\ Processes and Engineering in \\ Mechanics and Materials Laboratory, \\ (PIMM, CNRS UMR 8006), \\ Arts \& Métiers ParisTech, \\ 151 boulevard de l'Hôpital, \\ Approach for Quasi-Periodic Motions and Stability Analysis}

75013 Paris, France

e-mail: mikhail.guskov@paris.ensam.fr

Fabrice Thouverez

Professor

Laboratory of Tribology and Dynamics of Systems,

(LTDS, CNRS UMR 5513),

Ecole Centrale de Lyon,

36 avenue Guy de Collongue,

69130 Ecully cedex, France

e-mail: fabrice.thouverez@ec-lyon.fr

\begin{abstract}
Quasi-periodic motions and their stability are addressed from the point of view of different harmonic balance-based approaches. Two numerical methods are used: a generalized multidimensional version of harmonic balance and a modification of a classical solution by harmonic balance. The application to the case of a nonlinear response of a Duffing oscillator under a bi-periodic excitation has allowed a comparison of computational costs and stability evaluation results. The solutions issued from both methods are close to one another and time marching tests showing a good agreement with the harmonic balance results confirm these nonlinear responses. Besides the overall adequacy verification, the observation comparisons would underline the fact that while the $2 D$ approach features better performance in resolution cost, the stability computation turns out to be of more interest to be conducted by the modified $1 D$ approach
\end{abstract}

\section{Introduction}

Multifrequency dynamic behavior is a feature of many technical systems. Difficulties arise when these systems feature nonlinear properties. This is typically the case of multiple input systems or systems with internal resonances, in particular, electronic circuits $[1,2]$ or mechanical systems such as multiple-rotor systems [3-6].

One of the fields of interest concerning these systems is their steady-state behavior. The direct time solution of related equation systems is often too costly. Several approaches exist to study the steady-state response of nonlinear systems: methods of multiple scales aiming the research of consecutive approximations to the exact solutions [7], methods of harmonic balance [8-13] providing a projection of the sought solution on the harmonics of a basis sine function, and shooting-type methods [14] seeking the initial condition located on a steady state trajectory of the full system. Once the periodic solution is found, its stability is quite straightforward to obtain through the classical Floquet monodromy matrix [15]. In 2006, Dunne and Hayward [16] presented the splitfrequency harmonic balance method. This approach offers an algorithmic optimization when the addressed systems are under periodic excitation composed of several frequency components, which are multiples of the fundamental one, in order to split the low- and high-frequency response processing.

Common methods of steady-state solutions are not valid when it comes to multifrequency systems with nonlinear properties undergoing quasi-periodic motion. However, by introducing the concept of the multidimensional time and frequency domain these techniques can be generalized.

In 1981, Chua and Ushida [1] presented a generalization of the harmonic balance method in application to multifrequency electrical circuits. Nonlinear terms have been processed by the trigonometrical collocation-type procedure. In 1983, Lau and Cheung [17] adjusted this method to an incremental harmonic balance formulation. This method was further developed by Kim and Noah in 1996 and 1997 by coupling it with an alternative timefrequency domain (AFT) approach and using the FFT $[18,19]$. In 2004, Pusenjak and Oblak [20] presented a multidimensional harmonic balance method with arc length continuation. The work of Schilder et al. in 2006 [2] has provided an overview of two var- iants of frequency-domain methods based on the averaging approach: semidiscretization and full-discretization, implying the FFT-processing over one single dimension or all of the dimensions of the torus representing the multidimensional period. In 2009, Akgün et al. [21] realized a symbolic algorithm for studying the quasi-periodic systems. A good agreement is observed with the time marching simulations, however, this algorithm is limited to nonlinearities allowing a symbolic Fourier transform. Several methodological issues have also been considered, as by Guskov et al. in 2008 [22], for semianalytical Jacobian computation approaches.

Recent works began showing applications of multidimensional harmonic balance to real mechanical systems. Namely, in 2005, Legrand [23] studied rotor-stator interactions in aircraft engines. In 2007, Guskov et al. [6] presented an analysis of a nonlinear dual rotor-bearing system under multiple unbalances excitations. In 2004, Laxalde et al. [24] addressed the case of excitation present in multistage bladed disk systems using the multidimensional time-frequency framework.

The stability study of quasi-periodic motions requires a specific approach. In 1985, Kaas-Petersen proposed and then developed a shooting-based approach, implying the use of higher order Poincaré maps, corresponding to a conceptual generalization of the monodromy matrix [25-27]. This approach was later applied and numerically improved by Kim $[18,28]$.

The present work presents a comparison between two frequency domain approaches in application to a Duffing oscillator under quasi-periodic excitation due to two incommensurate excitation frequencies. The approaches we have used are a generalized multidimensional harmonic balance technique and a classical harmonic balance used with a rounded ratio between the excitation component frequencies. In the following, the first technique will be denoted as the multidimensional harmonic balance method (MHBM) while the second one will be called the adjusted harmonic balance method (AHBM). These two methods are compared, especially from the practical point of view, such as, for instance, algorithmic issues or a stability study. In particular, a simplified approach to the stability evaluation of quasi-periodic motions is elaborated based on the AHBM.

\section{Numerical Method: Generalized Harmonic Balance}

The systems addressed in this study are derived from the system of simultaneous second order differential equations, such as for structural dynamics, leading to the following matrix equation of size $n$ 


$$
\mathbf{M} \ddot{\mathbf{x}}+\mathbf{D} \dot{\mathbf{x}}+\mathbf{K} \mathbf{x}+\mathbf{f}_{\mathrm{NL}}(\mathbf{x})=\mathbf{g}(t)
$$

where $\mathbf{M}, \mathbf{D}$, and $\mathbf{K}$ stand for mass, generalized damping, and stiffness matrices, respectively, $\mathbf{x}, \mathbf{f}_{\mathbf{N L}}$, and $\mathbf{g}$ denote generalized displacements, nonlinear terms, and excitation vectors, respectively, and $n$ is the number of degrees of freedom.

Mechanical systems are often subject to a periodic excitation which may be represented as a Fourier series

$$
\mathbf{g}(t)=\sum_{k=0}^{\infty} \tilde{\mathbf{c}}_{k} \cos k \omega t+\tilde{\mathbf{d}}_{k} \sin k \omega t
$$

The steady-state response to such excitation is often sought in an analogous form

$$
\mathbf{x}(t)=\sum_{k=0}^{\infty} \tilde{\mathbf{a}}_{k} \cos k \omega t+\tilde{\mathbf{b}}_{k} \sin k \omega t
$$

Here, the tilde sign $\left(^{\sim}\right)$ is used to denote the frequency domain variables. The presence of a particular frequency makes it interesting to introduce a normalized time variable

$$
\tau=\omega t
$$

A number of applied methods exists for extracting motions of the form (3); the harmonic balance is one of the most widespread.

The numerical method of this work is based on the classical harmonic balance method; a summary of this approach is first given in order to introduce main concepts. The modifications of this method are then realized in order to adjust them to the addressed problematics.

2.1 Classical Harmonic Balance. In the following, we will hold to the notations analogous to those of Puseniak et al. [20]. Thus, the inverse descrete Fourier transform (2) will be denoted by means of the matrix $\mathbf{T}(\tau)$ composed of sines and cosines up to order $N$, which produces $N_{H}=2 N+1$ harmonic components

$$
\mathbf{x}(\tau)=\mathbf{T}(\tau) \tilde{\mathbf{x}}
$$

where the vector $\tilde{\mathbf{x}}$ ( size, $\left.N_{H} n\right)$ is composed of the components of $\tilde{\mathbf{a}}$ and $\tilde{\mathbf{b}}$ in accordance with $\mathbf{T}$.

The substitution of the approximation (5) in the equation of motion (1) yields

$$
\omega^{2} \mathbf{M} \frac{\partial^{2} \mathbf{T}}{\partial \tau^{2}} \tilde{\mathbf{x}}+\omega \mathbf{D} \frac{\partial \mathbf{T}}{\partial \tau} \tilde{\mathbf{x}}+\mathbf{K T} \tilde{\mathbf{x}}+\mathbf{f}_{\mathrm{NL}}(\mathbf{T} \tilde{\mathbf{x}})-\mathbf{T} \tilde{\mathbf{g}}=0
$$

To eliminate the time variable, the functions defined by Eq. (6) can be projected on the same trigonometric basis given by the $\mathbf{T}$ matrix

$$
\int_{0}^{2 \pi} \mathbf{T}^{\mathrm{T}}\left(\omega^{2} \mathbf{M} \frac{\partial^{2} \mathbf{T}}{\partial \tau^{2}} \tilde{\mathbf{x}}+\omega \mathbf{D} \frac{\partial \mathbf{T}}{\partial \tau} \tilde{\mathbf{x}}+\mathbf{K} \mathbf{T} \tilde{\mathbf{x}}+\mathbf{f}_{\mathrm{NL}}(\mathbf{T} \tilde{\mathbf{x}})-\mathbf{T} \tilde{\mathbf{g}}\right) d \tau=0
$$

This corresponds to a Galerkin-type procedure with trigonometrical functions constituting $\mathbf{T}$ simultaneously used as trial and weight functions. With this choice, the periodicity boundary conditions are automatically satisfied.

The projection (7) results in a system of nonlinear algebraic equations with $\tilde{\mathbf{x}}$ as unknowns

$$
\mathbf{L} \tilde{\mathbf{x}}+\tilde{\mathbf{f}}_{\mathrm{NL}}(\tilde{\mathbf{x}})-\tilde{\mathbf{g}}=\tilde{\mathbf{r}}(=0)
$$

Here, $\mathbf{L}$ stands for the linear part of the equation (dynamic stiffness corresponding to each harmonic component)

$$
\begin{aligned}
& \mathbf{L}=\int_{0}^{2 \pi} \mathbf{T}^{\mathrm{T}}\left(\omega^{2} \mathbf{M} \frac{\partial^{2} \mathbf{T}}{\partial \tau^{2}}+\omega D \frac{\partial \mathbf{T}}{\partial \tau}+\mathbf{K T}\right) d \tau \\
&= {\left[\begin{array}{llll}
\mathbf{L}^{\{0\}} & 0 & \ldots & 0 \\
0 & \mathbf{L}^{\{1\}} & \ldots & 0 \\
\vdots & \vdots & \ddots & \vdots \\
0 & 0 & \ldots & \mathbf{L}^{\{N\}}
\end{array}\right] } \\
& \mathbf{L}^{\{0\}}=\mathbf{K} ; \quad \mathbf{L}^{\{j\}}=\left[\begin{array}{ll}
\mathbf{K}-(j \omega)^{2} \mathbf{M} & j \omega \mathbf{D} \\
-j \omega \mathbf{D} & \mathbf{K}-(j \omega)^{2} \mathbf{M}
\end{array}\right], j=1, \ldots N
\end{aligned}
$$

$\tilde{\mathbf{g}}$ and $\tilde{\mathbf{f}}$ correspond to excitation and nonlinear terms

$$
\tilde{\mathbf{g}}=\int_{0}^{2 \pi} \mathbf{T}^{\mathrm{T}} \mathbf{g} d \tau, \quad \tilde{\mathbf{f}}_{\mathrm{NL}}(\tilde{\mathbf{x}})=\int_{0}^{2 \pi} \mathbf{T}^{\mathrm{T}} \mathbf{f}_{\mathbf{N L}}(\mathbf{T} \tilde{\mathbf{x}}) d \tau
$$

and $\tilde{\mathbf{r}}$ is the associated residual function. The nonlinear algebraic equation (8) can be solved by a Newton-like method.

This approach, limited to the periodic motions, can be extended to a broader class of dynamical problems; in particular, to the cases of multiple excitations when the frequencies are not multiples of one another. We will consider the case of the steady response of nonlinear systems to two frequency components of excitation $\omega_{1}$ and $\omega_{2}$ with the frequency ratio

$$
\eta=\frac{\omega_{1}}{\omega_{2}}
$$

which is not rational, thus disabling the direct use of HBM. Two methods of the generalization of the harmonic balance are considered in this work. The first one consists of a direct generalization of the Galerkin approach to a two-dimensional time domain. The second approach would involve an approximation $\left(\tilde{\omega}_{1}, \tilde{\omega}_{2}\right)$ of the excitation frequencies in order to obtain a mono-dimensional frequency domain with the basic frequency $\omega_{0}$ which would be the greatest common divisor of $\tilde{\omega}_{1}$ and $\tilde{\omega}_{2}$ in which the excitation terms would correspond to integer harmonics.

2.2 Multidimensional Time and Frequency Domain. Many types of technical systems are known to have such a dynamical behavior that can be represented by trigonometric polynomials with $M>1$ basic frequencies

$\mathbf{x}(t)=\sum_{\mathbf{k} \in \mathbb{Z}^{M}}^{\infty} \tilde{\mathbf{a}}_{\mathbf{k}} \cos (\mathbf{k}, \boldsymbol{\omega}) t+\tilde{\mathbf{b}}_{\mathbf{k}} \sin (\mathbf{k}, \boldsymbol{\omega}) t, \quad \boldsymbol{\omega}=\left[\omega_{1}, \ldots, \omega_{M}\right]^{T} \in \mathbb{R}^{M}$

Here, the vector $\boldsymbol{\omega}$ is the frequency basis, $\mathbf{k}=\left[k_{1}, \ldots, k_{M}\right]$ stands for the vectorial harmonic indexes and (,) stands for the dot product

$$
(\mathbf{k}, \omega) t=\sum_{j=1}^{M} k_{j} \omega_{j} t
$$

One of the approaches to such functions is to consider $\mathbf{x}(t)$ as an equivalent function $\overline{\mathbf{x}}(\tau)$ of $M$ time variables in a $M$-dimensional time domain $\tau=\left[\tau_{1}, \ldots, \tau_{M}\right]=\boldsymbol{\omega} t, 2 \pi$-periodic in each component of the hyper-time $\tau$

$$
\mathbf{x}(t)=\overline{\mathbf{x}}(\tau)=\overline{\mathbf{x}}(\omega t)
$$

The time derivatives involved in such hyper-time variables are reformulated as follows

$$
\dot{\mathbf{f}}=\frac{\partial \mathbf{f}}{\partial t}=\frac{\partial \overline{\mathbf{f}}}{\partial \tau} \frac{\partial \tau}{\partial t}=\frac{\partial \overline{\mathbf{f}}}{\partial \tau} \omega
$$




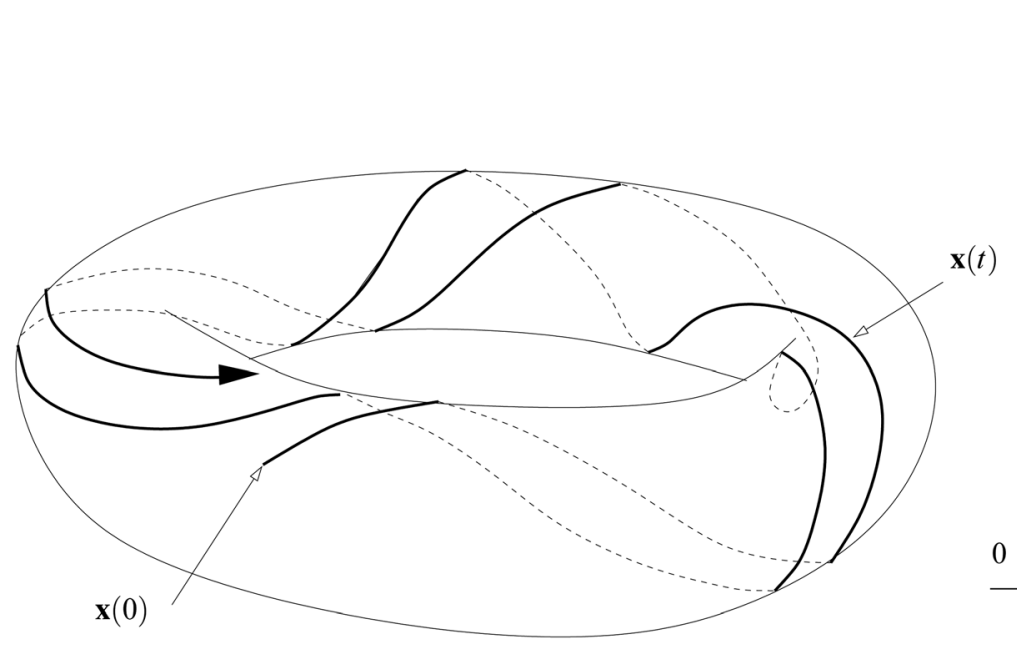

(a) Bi-periodic motion on a torus

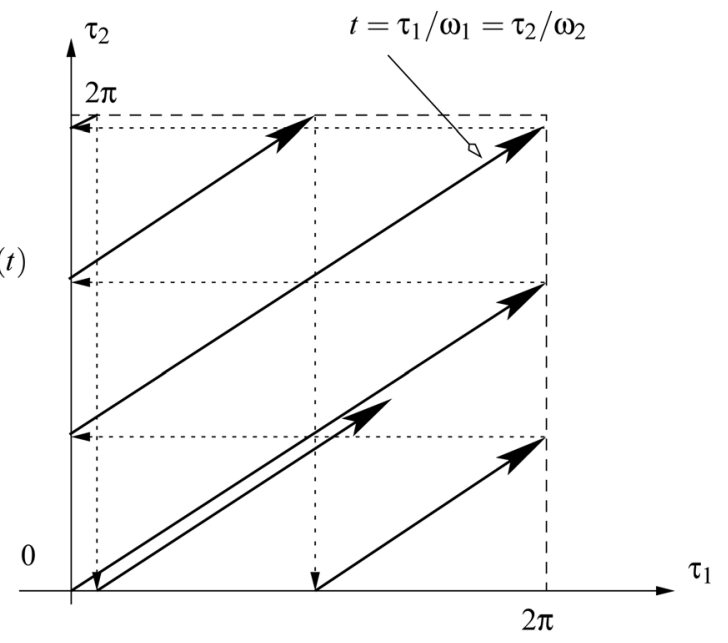

(b) Bi-dimensional time domain

Fig. 1 Multidimensional time domain concept

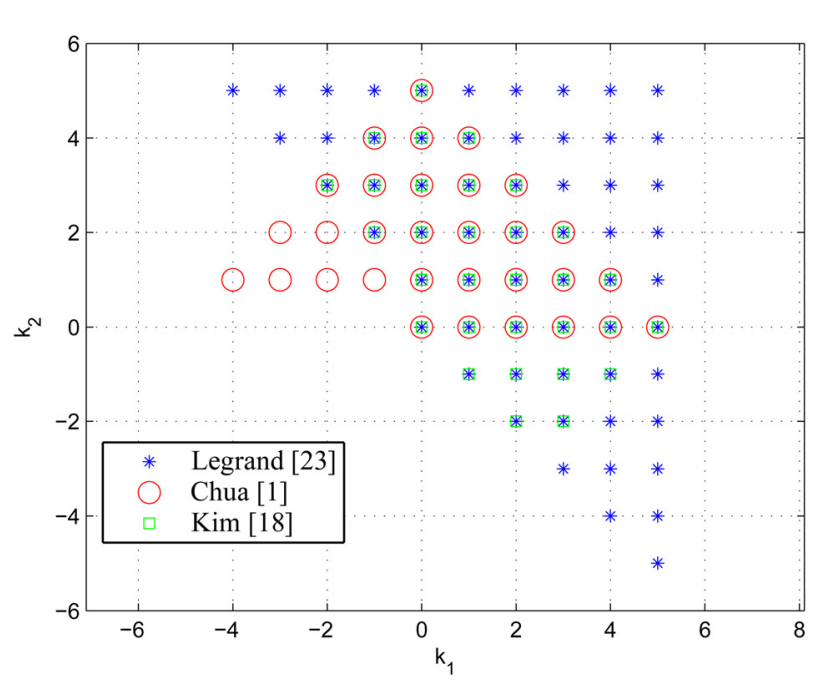

Fig. 2 Harmonic indices sets $(M=2, N=5)$

As shown by Schilder [2] in the case of $M=2$, a change of variables allows us to display the bi-periodic motion (13) as covering an invariant torus, which is schematically represented in Fig. 1(a). An unfolded view of the associated time domain is given in Fig. 1(b).

To clarify the presentation, the bar sign referring to the multidimensional time domain will be omitted in the following. This generalization may also be performed with the series representation (3). The truncation to the harmonic $N$ is carried out in several ways. Different examples of the set of truncated indexes for $M=2$ and $N=5$ are shown in Fig. 2 . In the present work, the third case is used, which corresponds to the following condition

$$
\sum_{i=1}^{M}\left|k_{i}\right| \leq N
$$

which implies the presence of $N_{H}$ harmonic terms

$$
N_{H}=\frac{(2 N+1)^{M}+1}{2}
$$

It is to be observed in Fig. 2 that only half of the terms from the range given by the inequality (17) are kept. This is due to the symmetry of the Fourier transform of real periodic functions.
The previously described harmonic balance method can be extended to multifrequency systems through a direct generalization, according to the multidimensional concepts.

2.3 Generalized Harmonic Balance. By substituting the harmonic representation (13) into the equation of motion (1) and by projecting it on the same harmonic functions by a Galerkin procedure analogous to Eq. (7), we obtain the harmonic balance equation similar to Eq. (8)

$$
\mathbf{L} \tilde{\mathbf{x}}+\tilde{\mathbf{f}}_{\mathrm{NL}}(\tilde{\mathbf{x}})-\tilde{\mathbf{g}}=\tilde{\mathbf{r}}(=0)
$$

where $\mathbf{L}$ is modified for the new harmonic contents considered

$$
\begin{gathered}
\mathbf{L}=\left[\begin{array}{llll}
\mathbf{L}^{\{0\}} & 0 & \ldots & 0 \\
0 & \mathbf{L}^{\left\{\mathrm{k}_{1}\right\}} & \ldots & 0 \\
\vdots & \vdots & \ddots & \vdots \\
0 & 0 & \ldots & \mathbf{L}^{\left\{\mathrm{k}_{N_{H}}\right\}}
\end{array}\right] \\
\mathbf{L}^{\{0\}}=\mathbf{K}, \mathbf{L}^{\left\{\mathbf{k}_{j}\right\}}=\left[\begin{array}{cc}
\mathbf{K}-\left(\mathbf{k}_{j}, \boldsymbol{\omega}\right)^{2} \mathbf{M} & \left(\mathbf{k}_{j}, \boldsymbol{\omega}\right) \mathbf{D} \\
-\left(\mathbf{k}_{j}, \boldsymbol{\omega}\right) \mathbf{D} & \mathbf{K}-\left(\mathbf{k}_{j}, \boldsymbol{\omega}\right)^{2} \mathbf{M}
\end{array}\right], \\
j=1, \ldots N_{H}-1
\end{gathered}
$$

The AFT approach can be generalized by the use of $M$-dimensional FFT in order to apply it to the $M$-dimensional hyper-time period.

2.4 Mono-Dimensional Approximation for Multiharmonic Problems. We will now develop an approach based on an approximation of the multifrequency problem for the case of $M=2$ by a system founded on the frequency $\omega_{0}$ which is the greatest common divisor of $\tilde{\omega}_{1}$ and $\tilde{\omega}_{2}$

$$
\omega_{0}=\frac{\tilde{\omega}_{i}}{p_{i}}, \quad i=1, \ldots, M, \quad p_{i} \in \mathbb{N}
$$

In the case of $M=2$, we have

$$
\frac{p_{1}}{p_{2}}=\tilde{\eta} \approx \eta
$$

providing a fair estimate of $\eta$ when possible, and $\left(\tilde{\omega}_{1}, \tilde{\omega}_{2}\right)$ provide an approximation of $\left(\omega_{1}, \omega_{2}\right)$. In the following, this approach will be denoted as the adjusted HBM (AHBM). 
Table 1 Harmonic terms number for MHBM and AHBM for the example frequency ratio (31) and (33)

\begin{tabular}{lrr}
\hline \hline$N$ & MHBM & AHBM \\
\hline 1 & 3 & 3 \\
3 & 13 & 13 \\
5 & 31 & 31 \\
7 & 57 & 57 \\
9 & 91 & 83 \\
11 & 133 & 103 \\
13 & 183 & 123 \\
\hline \hline
\end{tabular}

For this method, the trigonometrical representation response $\mathbf{x}$ would differ from Eq. (3)

$$
\begin{aligned}
\mathbf{x}(t)= & \sum_{\left(k_{1}, k_{2}\right) \in \mathbb{Z}_{N}^{2}}^{\infty} \tilde{\mathbf{a}}_{k_{1} k_{2}} \cos \left(k_{1} p_{1}+k_{2} p_{2}\right) \omega_{0} t \\
& +\tilde{\mathbf{b}}_{k_{1} k_{2}} \sin \left(k_{1} p_{1}+k_{2} p_{2}\right) \omega_{0} t
\end{aligned}
$$

because the spectrum of the motion sought by this method would not necessarily contain all the components $k \omega_{0}$ with $k \in \mathbb{N}_{p N}$ for some given $N$ with $\max \left(p_{1}, p_{2}\right)$. Only those corresponding to the combinations of the excitation terms $\left(k_{1} p_{1}+k_{2} p_{2}\right) \omega_{0}$ with $\left(k_{1}, k_{2}\right) \in \mathbb{Z}_{N}^{2}$ would be considered. Furthermore, in the case of the rational approximation (23) [several terms of the decomposition may be superimposed and thus only one of these terms is kept. This issue is illustrated in the example below; see Table 1 and Fig. 4.

This issue can also be interpreted in terms of small denominators arising in the case of multiple frequency systems, which is mentioned by Kaas-Petersen in the context of KolmogorovArnold-Moser theory [27]. The approximation of the frequency basis by a set of frequencies admitting a rational proportion with a relatively small denominator makes the consideration of the higher terms of the multiple Fourier series inaccessible. Strictly speaking, the numerical representation of an irrational frequency ratio, used in the MHBM approach is also necessarily rational, however, the error thus induced is far below the sensitivity of the computation, especially that of the discrete Fourier transform. Therefore, to approach the limitations of such a representation, one would require a very large number of harmonic terms to take into account and, correspondingly, a very large number of points in the time discretization.

2.5 Path Following: Arc Length Continuation. To ensure the proper path following on the response curve, an arc length continuation similar to that of [20] is used. It consists of controlling the length of the curvilinear step $\Delta s$ along the response curve $\tilde{\mathbf{x}}(\nu)$. To this end, the solution of the nonlinear equation (19) is coupled with an arc length continuation condition

$$
\left\|\left[\begin{array}{l}
\mathbf{x} \\
\nu
\end{array}\right]^{j+1}-\left[\begin{array}{c}
\tilde{\mathbf{x}} \\
\nu
\end{array}\right]^{j}\right\|^{2}-\Delta s^{2}=0
$$

Here, $\nu$ is the control parameter and the superscripts $j$ and $j+1$ denote the number of considered consecutive points on the response curve. The step size $\Delta s$ is adjusted according to the convergence of previous steps.

The expression (25) provides an additional equation to the system (19) so that one can determine the value of $\nu$. In our study, the control parameter is equal to $\omega_{1}$.

\section{Stability}

Usually, the stability of periodic motions is studied by using the Floquet theory, which consists of computing the eigenspectrum of the monodromy matrix. The latter expresses whether the Poincaré map is contracting (asymptotic stability) [7]. This approach, suitable for the AHBM periodic approximation, is first presented. This would also allow us to introduce the terms required for the second-order Poincaré map description, used for the bi-periodic motions stability study $[18,25,29]$, as they are issued from the MHBM.

Usually, the Floquet monodromy matrix is computed from the integration of the perturbation system [7]

$$
\dot{\mathbf{y}}=\mathbf{B y}
$$

over the time period $[0, T]$. Here, $\mathbf{y}$ is the state-space representation of the perturbation motion around the reference solution $\mathbf{x}^{o}$ being tested and $\mathbf{B}$ is the associated matrix of the perturbation system

$$
\mathbf{y}=\left[\begin{array}{c}
\mathbf{x}-\mathbf{x}^{o} \\
\dot{\mathbf{x}}-\dot{\mathbf{x}}^{o}
\end{array}\right], \quad \mathbf{B}=\left(\begin{array}{ccc}
0 & \mathbf{I} \\
\mathbf{M}^{-1}\left(\mathbf{K}+\left[\frac{\partial \mathbf{f}_{\mathbf{N K}}}{\partial \mathbf{x}}\right]_{\mathbf{x}^{o}}\right) & \mathbf{M}^{-1} \mathbf{D}
\end{array}\right)
$$

Such an integration is conducted for $2 n$ linearly independent initial conditions $\mathbf{y}_{0 i}$ thus providing a basis $\left\{\mathbf{y}_{i}\right\}$ in the space of solutions of Eq. (26). This basis is grouped in the matrix

$$
\Phi(t)=\left[\mathbf{y}_{1}(t), \ldots, \mathbf{y}_{2 n}(t)\right]
$$

called the fundamental matrix. The initial conditions are commonly chosen in order to have $\Phi(0)=I$. The monodromy matrix itself is defined as the matrix of transition over the period $T$

$$
\mathbf{Q}=\Phi(T) \Phi(0)^{-1}
$$

The spectrum of eigenvalues of $\mathbf{Q}$ called multipliers determine the stability of the studied solution.

In the case of the AHBM approach, the system has a period $T_{o}=2 \pi / \omega_{o}$ and the conventional Floquet method may be applied. In the quasi-periodic case, the period $T$ is not available. However, a matrix analogous to $\mathbf{Q}$ may be defined in the framework of the multidimensional representation. Figure 3 gives a schematic overview of an approach based on an extension of the Poincaré section concept. This generalization consists of the interpolation of the Floquet transition matrix for the perturbed system, sampled over the instants $\mathrm{t}_{q(1)}=q T_{1}$ with the $T_{1}=2 \pi q / \omega_{1}$ period with respect to the first time scale, which yields the Poincaré map $\mathcal{P}_{1}$ based on the section of the trajectory by the plane $\Sigma_{1}\left(\tau_{1}=0\right)$, as shown in Fig. 3(a), thus furnishing an approximation of the torus section by $\Sigma_{1}$ (Fig. 3(b)). An interpolation over the named points would provide an approximation of the value of the limit of this matrix in $t_{1(2)}=2 \pi \omega_{2}$, which would correspond to a full cycle over the second time dimension and have the stability properties analogous to those of the monodromy matrix. The procedure thus consists of approximating the section by the plane $\Sigma_{2}\left(\tau_{2}=0\right)$ of the trajectory's section by $\Sigma_{1}$ (see Fig. 3(c)). The interpolation of $\mathcal{P}_{2}$ is illustrated in Fig. $3(d)$. Indeed, despite the impossibility of the direct return to the starting point, the limit value of the transition matrix can be reconstructed by interpolation over the points near the start, over the values $\mathbf{Q}_{k}=\Phi\left(k T_{1}\right) \Phi(0)^{-1}$ [25]. These instants provide a set of trajectory points corresponding to the first periodicity $\left(\tau_{1 k}=2 \pi k\right.$, phase $\left.\phi_{1 k}=0\right)$ and to different phases with respect to the second periodicity component $\left(\tau_{2 k}=2 \pi k \eta\right.$, phase $\left.\phi_{2 k}=\tau_{2} k \bmod 2 \pi\right)$. Interpolating these values $\mathbf{Q}_{k}$ one obtains an approximation of the second order Poincaré map associated with the quasi-periodic motion under consideration. The spectrum of this matrix would determine the contracting properties of the mapping [18]. This procedure requires a very long time integration to achieve convergence. For instance, in the followingexample, for the AHBM, $T_{o}=10 T_{1}=7 T_{2}$, whereas for MHBM the integration time providing convergence for the interpolated monodromy 


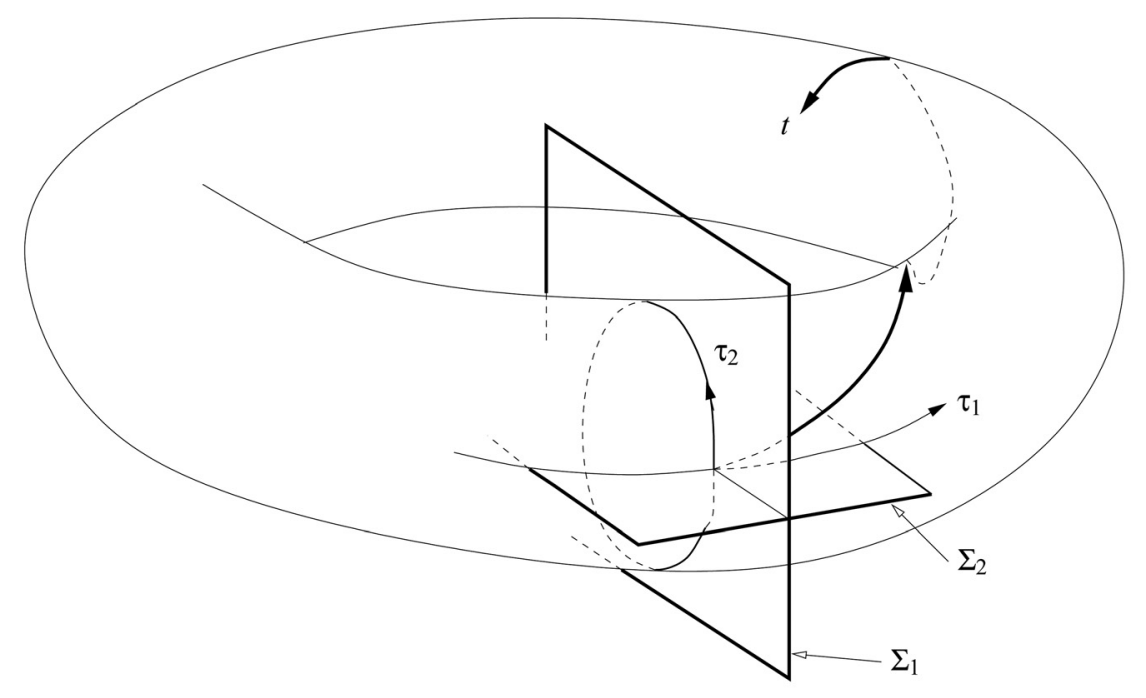

(a) Trajectory sections on the invariant torus by the planes $\tau_{1}=0$ et $\tau_{2}=0$

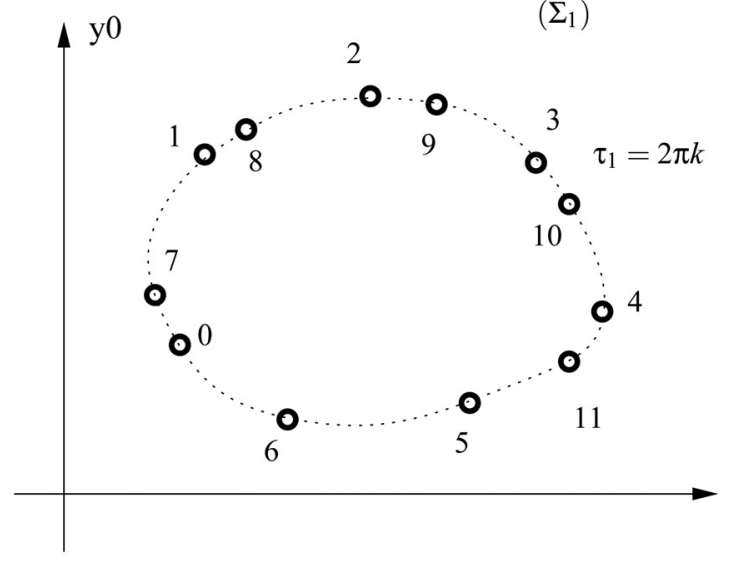

(b) Quasi-periodical trajectory Poincaré section

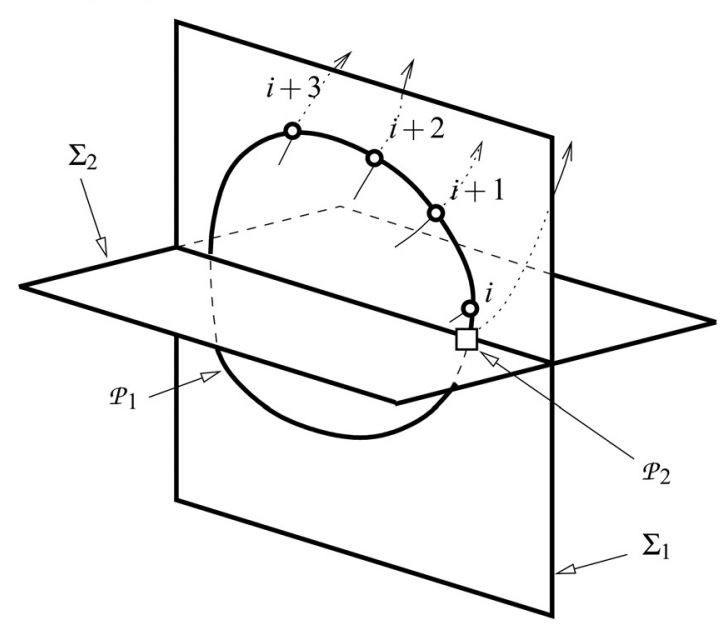

(c) Second order Poincaré section

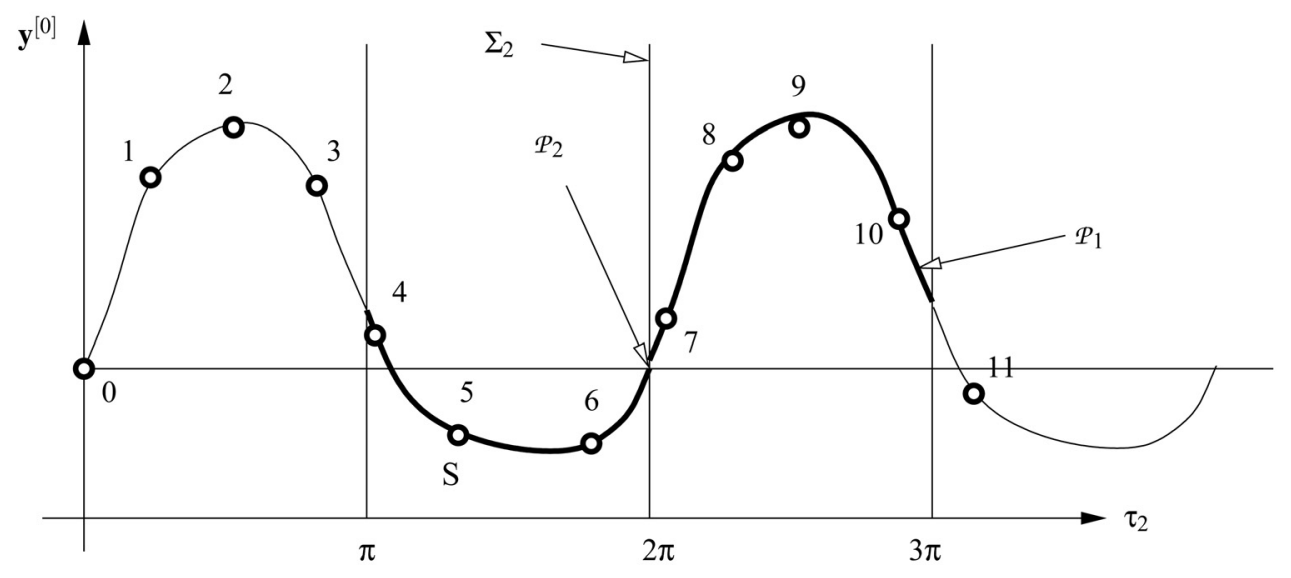

(d) Poincaré section interpolation

Fig. 3 Second order Poincaré section computation schematic

matrix amounts to $100 . .300 T_{1}$. Thus, although the number of evaluated fundamental solutions (28) is the same for both frameworks, the second order analysis turns out to be much more time consuming.

\section{Example: Duffing Oscillator}

The application is carried out on a bi-periodically excited Duffing oscillator, defined by the following equation 

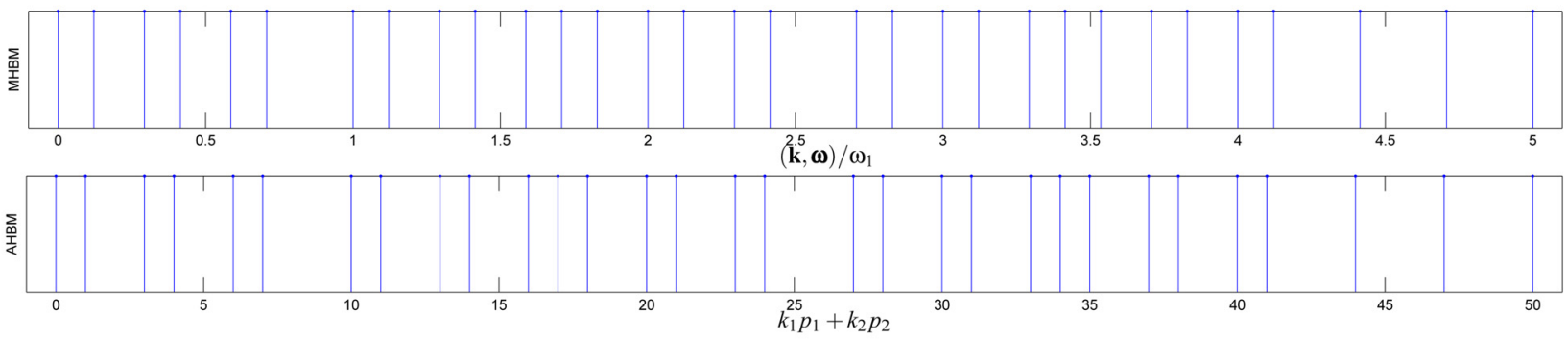

(a) $N=5$
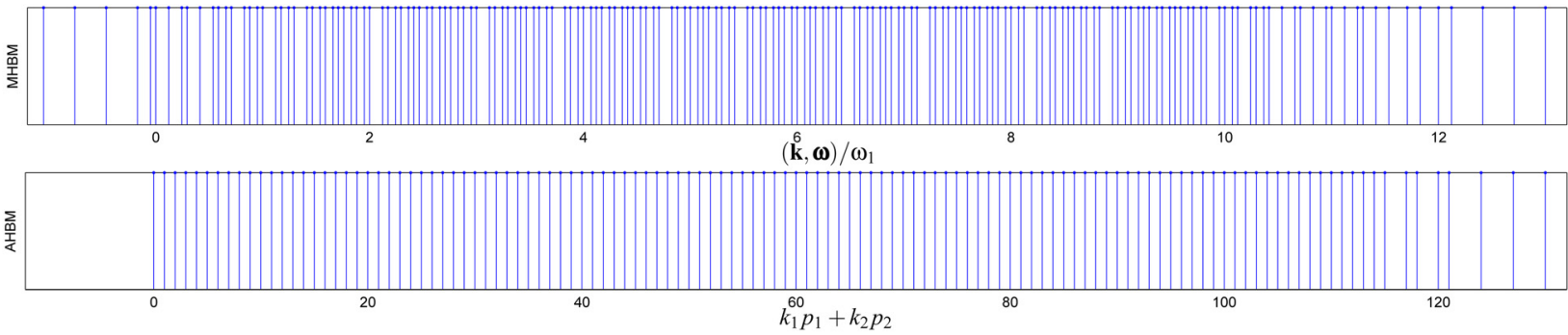

(b) $N=13$

Fig. 4 Harmonic terms frequency distribution for MHBM and AHBM for the example frequency ratio in Eqs. (31) and (33)

Table 2 CPU time comparison between MHBMi (FFT 2D over $i$ points w.r.t. each time scale) and AHBMi (FFT over $i$ points) for the example frequency ratio (31) and (33): mean computation time per point of the response curve calculated with the use of MATLAB

\begin{tabular}{lcccc}
\hline \hline$N$ & MHBM256 & MHBM32 & AHBM512 & AHBM1024 \\
\hline 1 & 2.15 & 0.065 & 1.42 & 8.92 \\
3 & 7.13 & 0.40 & 5.36 & 25.1 \\
5 & 18.4 & 1.92 & 12.2 & 61.94 \\
7 & 31.7 & 5.70 & 26.3 & 117.3 \\
9 & 54.7 & 13.6 & 42.9 & 220.3 \\
11 & 144.3 & 36.8 & 86.4 & 246.8 \\
13 & 260.6 & 63.4 & 150.9 & 367.9 \\
\hline \hline
\end{tabular}

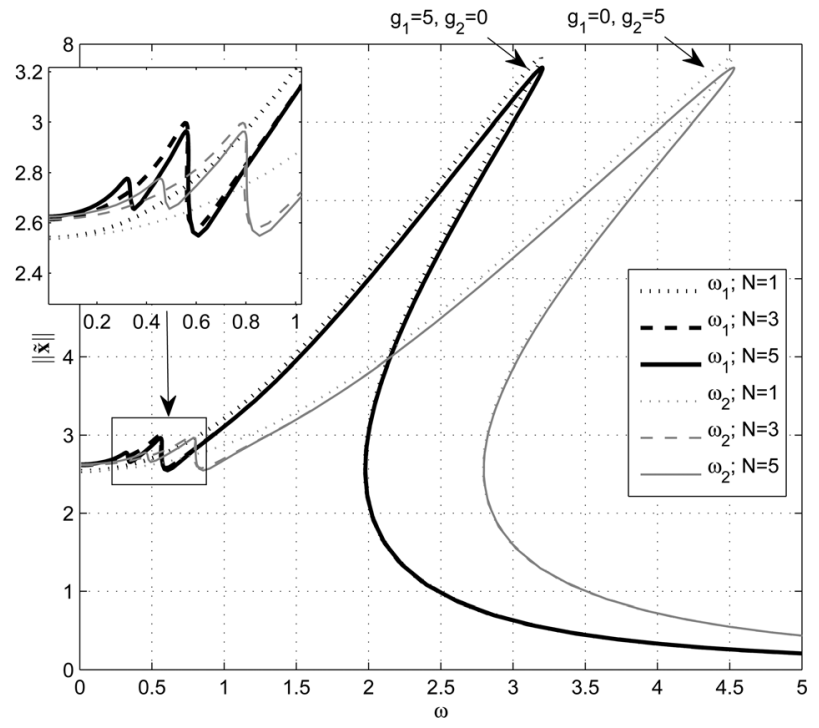

Fig. 5 Response of the Duffing oscillator (Eq. (30)) to each harmonic component of the excitation taken separately (Euclidean norm of the harmonic solution $\tilde{\mathbf{x}}$ )

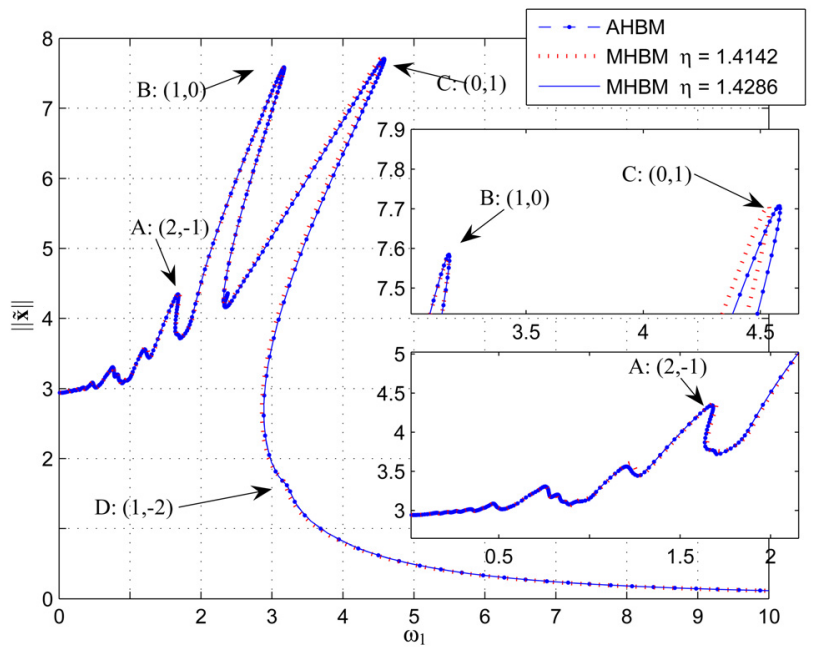

Fig. 6 Response curves for $N=13$ by the AHBM and MHBM

$$
\ddot{x}+2 \zeta \dot{x}+x+\gamma x^{3}=g_{1} \sin \omega_{1} t+g_{2} \sin \omega_{2} t
$$

with incommensurate frequencies of the excitation components

$$
\frac{\omega_{1}}{\omega_{2}}=\sqrt{2} \approx 1.4142
$$

and the following values for the system's parameter

$$
\zeta=0.1, \quad \gamma=0.2, \quad g_{1}=g_{2}=5
$$

The system (30) has been solved by multidimensional harmonic balance up to $N=13$ in order to obtain a solution which is converged with respect to $N$ over a significant part of the $\omega$ range. An adjusted harmonic balance (AHBM) solution has also been computed for an approximation to the frequency ratio (31)

$$
\frac{\tilde{\omega}_{1}}{\tilde{\omega}_{2}}=\frac{10}{7} \approx 1.4286
$$



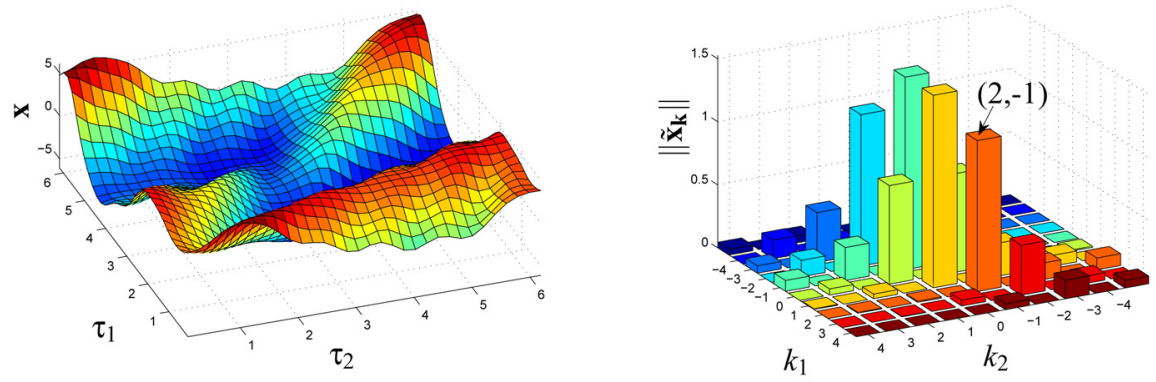

(a) Response at the resonance $\mathrm{A}\left(\omega_{1}=1.678,\|\tilde{\mathbf{x}}\|=4.343\right)$
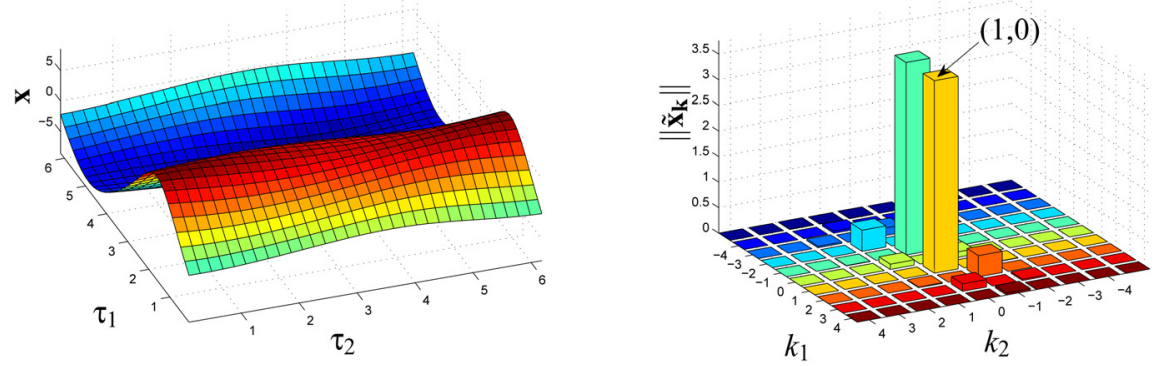

(b) Response at the resonance $\mathrm{B}\left(\omega_{1}=3.170,\|\tilde{\mathbf{x}}\|=7.584\right)$
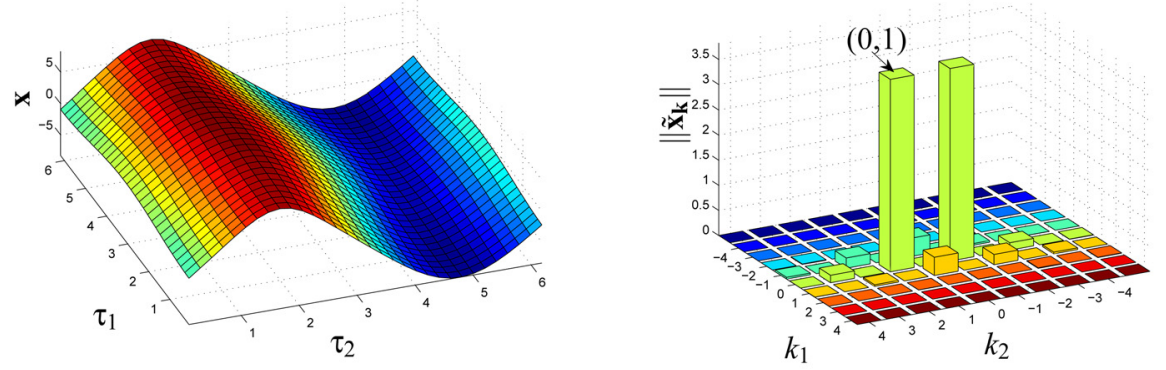

(c) Response at the resonance $C\left(\omega_{1}=4.582,\|\tilde{\mathbf{x}}\|=7.706\right)$
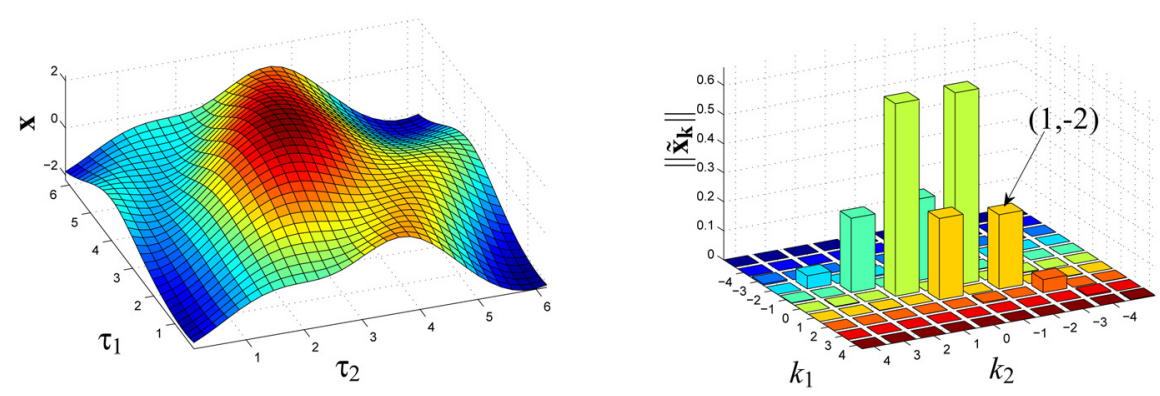

(d) Response at the resonance D $\left(\omega_{1}=3.257,\|\tilde{\mathbf{x}}\|=4.582\right)$

Fig. 7 Response of the Duffing oscillator (Eq. (30)) to a bi-periodic excitation on the resonance peaks : 2D time and frequency representation

which corresponds to a $1 \%$ accuracy and yields $\omega_{0}$ $=0.1 \omega_{1}=0.14286 \omega_{2}$. The number of harmonic terms involved in each case is presented in Table 1 . This quantity corresponds to the number of unknowns in the nonlinear equation system (19). As illustrated by Fig. 4, for small $N$ (e.g., $N=5$, Fig. 4(a)), the harmonic contents used by both methods are similar and have the same size. For higher $N$, the AHBM frequency components tend to fill the frequency domain uniformly (see Fig. 4(b)), which dif- fers from the MHBM case. The reduction of the number of terms in the AHBM computations is due to the frequency coincidence occurring between some components, such as, for instance, between $\mathbf{k}=(-3,8)$ and $\mathbf{k}=(4,-2)$, both corresponding to the frequency $\omega=(-3 \times 10+8 \times 7) \omega_{0}=(4 \times 10-2 \times 7) \omega_{0}=26 \omega_{0}$. One of these terms is redundant and thus, eliminated from consideration.

The AHBM strategy benefits from a simpler theoretical basis, although it requires a higher number of FFT points due to a small 


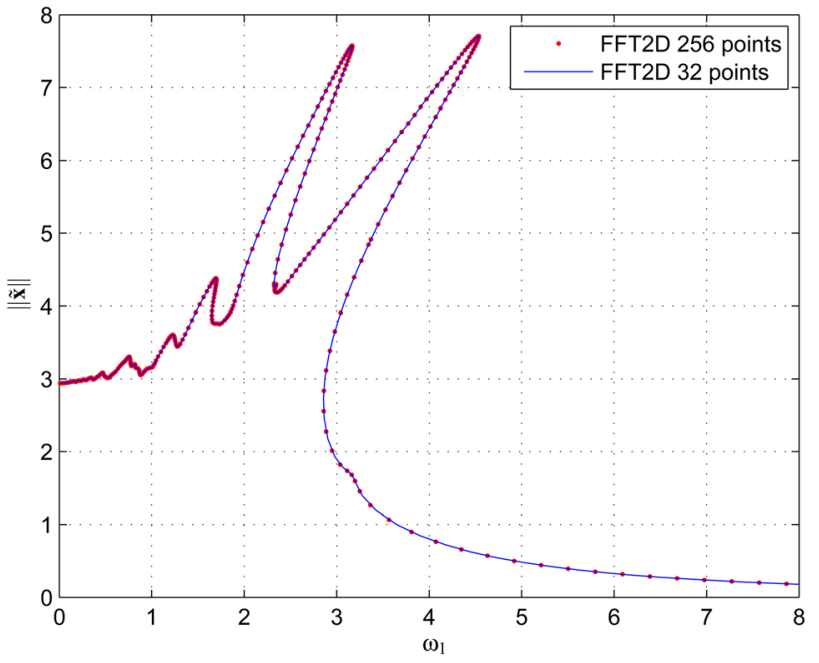

(a) Response curves for 32 and 256 points FFT sampling

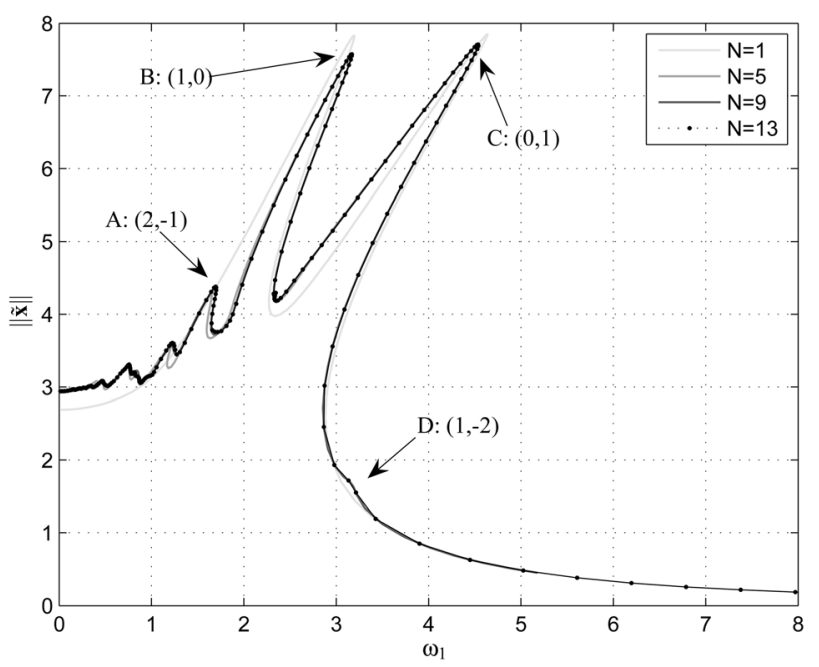

(b) Response curves for various $N$, important harmonic components indicated for principal resonance peaks

Fig. 8 Response of the Duffing oscillator (Eq. (30)) to a bi-periodic excitation: MHBM convergence study

$\omega_{0}$. Starting from $N=9$, the number of terms used in the MHBM exceeds that of the AHBM. This involves a reduction of the computational cost for the nonlinear solution, as shown in Table 2. However, the computational cost for the MHBM quasi-periodic solution can be significantly reduced by making a coarser time discretization to 32 points w.r.t. each time scale.

The response of the Duffing oscillator (30) to each component of the excitation taken separately is shown in Fig. 5. The plots feature the principal resonance peak and the superharmonic resonances of order 3 and 5. Naturally, there is a strict similarity between the response to each excitation component.

Figure 6 shows the response curves of the system (30) with both excitation terms simultaneously taken into account. The resonance peaks of the lowest order, i.e., for the components $\mathbf{k}=(2,-1),(1,0),(0,1),(1,-2)$ are highlighted as A, B, C, and D, respectively. The overall dynamical response magnitudes are in very good agreement between the two methods, although a slight frequency offset $(\approx 1 \%)$ is observed for the second peak, due to the excitation frequencies ratio discrepancy. This difference is absent when comparing the AHBM and MHBM using the same $\eta=1.4286$.

The behavior of the solution on these resonance peaks A, B, C, and D is illustrated in Fig. 7, showing the system's evolution in the $2 \mathrm{D}$ time domain and its $2 \mathrm{D}$ spectrum. In general, these plots feature periodicity with respect to each time dimension. On the

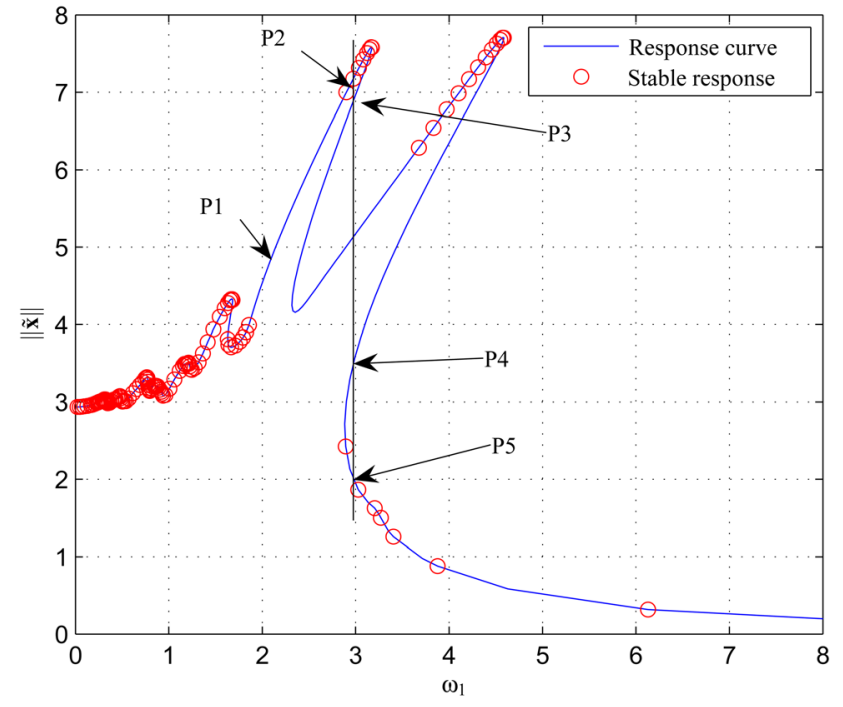

(a) Solution by AHBM, stability analysis by $1 \mathrm{D}$ Floquet approach

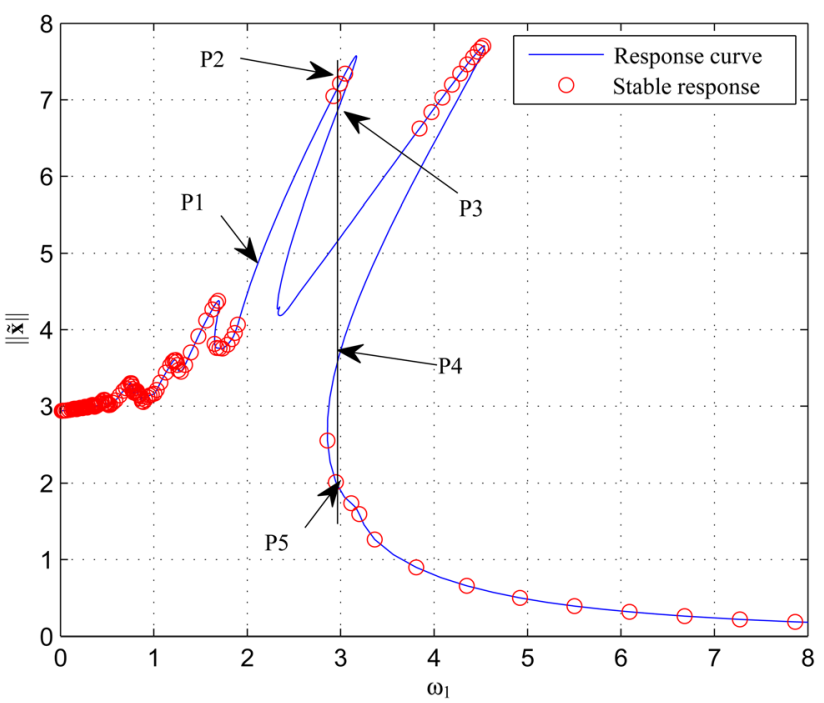

(b) Solution by MHBM, stability analysis by 2D Floquet approach

Fig. 9 Stability results for the Duffing oscillator (Eq. (30)) under bi-harmonic excitation (- response curve, $\circ$ stable)

principal peaks (Figs. 7(b) and 7(c)), the fundamental harmonic of the respective excitation term is dominant in the Fourier decomposition. The secondary peaks feature a significant contribution of higher harmonics, namely $(2,-1)$ for A (Fig. $7(a))$ and $(1,-2)$ for D (Fig. 7(d)).

As one may notice from Fig. 8(a), the 32 and 256 2D FFT points sampling yields the same response curve, which means that 32 points are sufficient and for finer sampling the MHBM is not sensitive to the time discretization. It is to be emphasized that when it comes to 2D FFT, the transform is computed with respect to both dimensions; thus, for instance, a 256 point $2 \mathrm{D}$ sampling corresponds to a double 256 point FFT over a $256 \times 256=65,536$ point $2 \mathrm{D}$ time domain. In a similar manner, the 32 point $2 \mathrm{D}$ discretization actually involves $32 \times 32=1024$ points, which explains why these results remain comparable to the AHBM solution based on 512 or 1024 FFT points.

The convergence, with respect to the harmonic order $N$ displayed in Fig. 8(b), shows that the response curves for $N=9$ and $N=13$ are superimposed and thus, $N=13$ corresponds to a converged solution.

A comparison of computational cost, depending on the chosen method, is given in Table 2. It should be noted that as long as the 

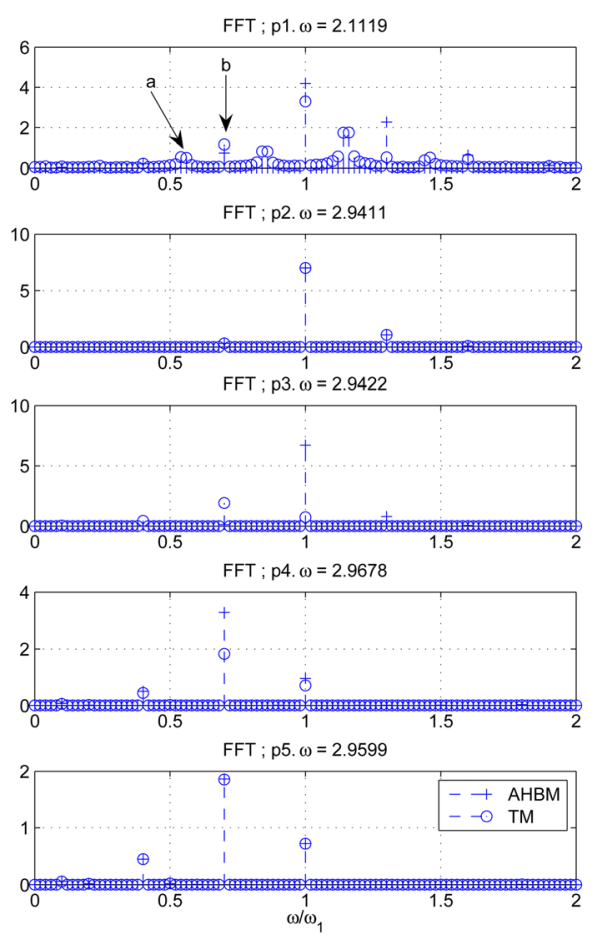

(a) AHBM
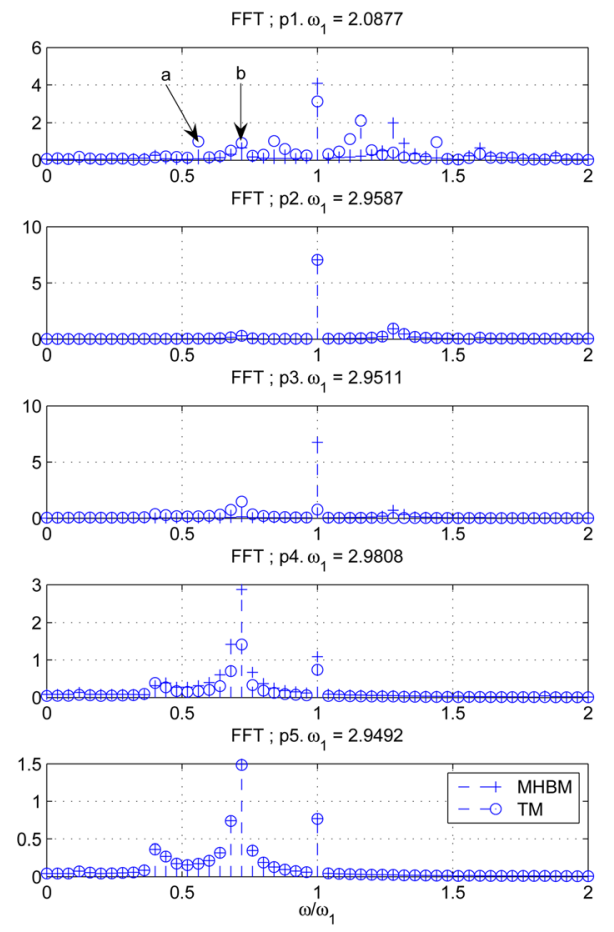

(b) MHBM
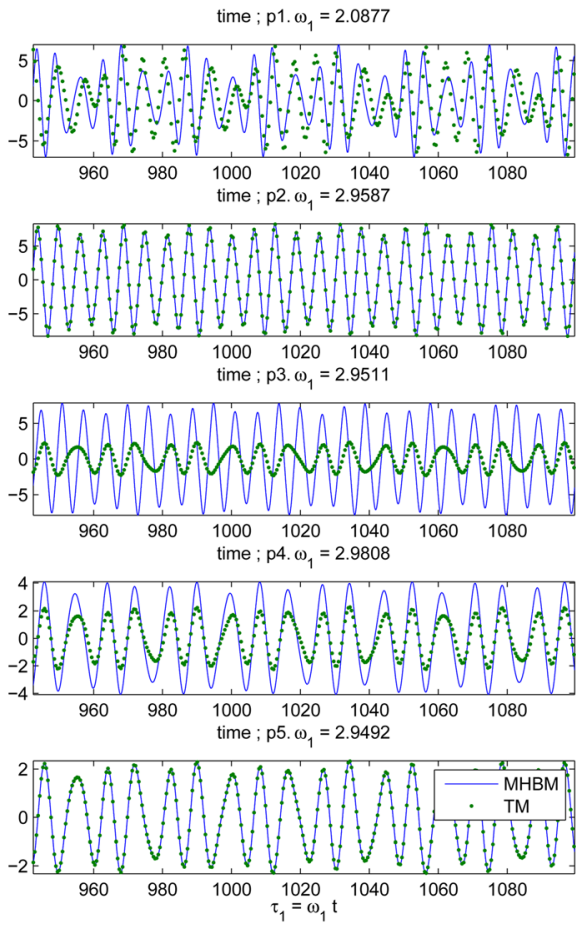

Fig. 10 Time marching (TM) test for several points of the response curve (Fig. 9) and respective FFT

sampling rate is similar, the calculation duration remains of the same order. However, the MHBM allows a much lower FFT discretization since the actual number of points used in the computation is the square of the discretization number, so it features a considerable advantage in the computation speed.

The stability results are presented in Fig. 9. Both methods yield quite similar results. Two features of these plots are of interest. First, the left sides of the main resonance peaks contain instability zones (such as in case of the first peak, $2.2<\omega_{1}<2.8$ ). These instabilities correspond to period-doubling-type bifurcations for both solution approaches. Although the results are similar, the AHBM 1D approach to the stability is more practical because of a much faster and simpler realization and the shorter time integration required.

Several characteristic points will now be discussed in order to illustrate the results in detail. These points are shown on the response curve in Fig. 9 as P1,..,P5. The point P1 is situated in an unstable zone. The points P2,..,P5 feature different responses 
the same excitation $\left(\omega_{1} \approx 2,95\right)$. The plots of the time marching (TM) tests for these points are presented in Fig. 10. The numbers of the considered points are given in Fig. 9. One can observe a general resemblance of the time marching results between the AHBM and the MHBM results. The points P2 and P5 feature a full coincidence between the MHBM and TM. The point P1 is situated in an instability region following a period-doubling bifurcation for the AHBM (Fig. 10(a)) or, for the quasi-periodic motion, torus doubling [2] bifurcation (Fig. 10(b)). Although the overall response level is similar for the HBM and TM results, one can notice, on the FFT for the point P1, that on the TM response some halffrequency components (points $a$ and $b$ ), with respect to the peaks computed by the HBM arise, as predicted by the stability analysis.

The TM response magnitude is close to that predicted by the MHBM, although the waveforms are different. This discrepancy, accompanied by the FFT plot showing different active frequency components, is in accordance with the stability evaluation. For the points $\mathrm{P} 3$ and $\mathrm{P} 4$, one can notice that while the HBM computations indicate some unstable solution, the TM realization is equal to the behavior at point P5, which corresponds to a stable motion for the considered value of $\omega$.

\section{Conclusion}

A generalized harmonic balance method using the AFT approach and an arc length continuation is developed. This method is applied to a nonlinear dynamical system under quasiperiodic excitation with an approximation by a classical harmonic balance solution. The response is quasi-periodic and strongly nonlinear. It puts forward the mutual influence of different components of resonance due to nonlinear coupling. Combination resonances are also observed on mixed components of the response.

The stability test method, based on a generalization of the Floquet approach, is applied to this problem and has allowed us to observe period-doubling-type bifurcations on the left slope of the principal resonance peaks. The simplified multiharmonic approach (AHBM), based on the approximation of the frequency basis by a mono-dimensional one, yields results similar to those of the MHBM. The computational cost for the resolution is much lower in the case of the MHBM. Nevertheless, for the stability evaluation, both methods yield quite similar results while the AHBM approach allows a classical Floquet multipliers analysis, which is faster and is also interesting because of the simplicity and, thus, reliability of the computational procedure.

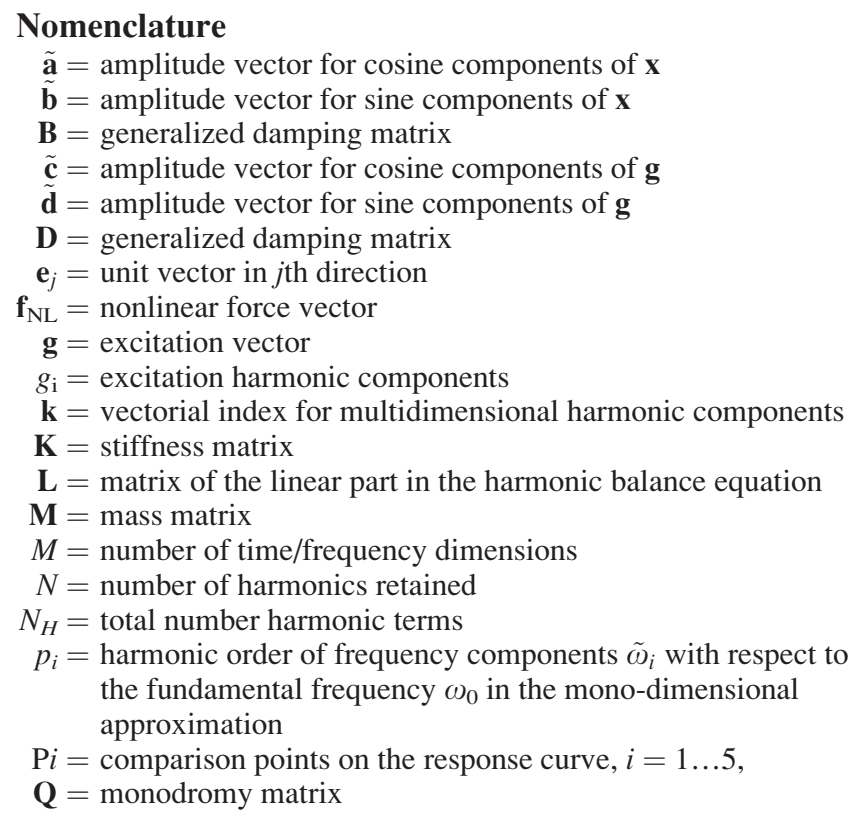

$\mathbf{r}=$ residual vector

$s=$ curvilinear co-ordinate along the response curve

$t=$ time

$T=$ time period

$\mathbf{T}=$ inverse discrete Fourier transform matrix

$\mathbf{x}=$ generalized displacement vector

$\mathbf{x}^{o}=$ reference motion in stability analysis

$\mathbf{y}=$ state-space representation of the perturbation around $\mathbf{x}^{o}$

$\gamma=$ nonlinear term coefficient in the Duffing oscillator example

$\zeta=$ damping parameter in the Duffing oscillator example

$\eta=$ frequency ratio

$\tilde{\eta}=$ rational approximation of frequency ratio

$\nu=$ control parameter

$\tau=$ dimensionless normalized time variable

$\tau=$ hyper-time vector

$\boldsymbol{\Phi}=$ fundamental matrix in stability analysis

$\omega=$ fundamental frequency in mono-dimensional case

$\boldsymbol{\omega}=$ frequency basis vector

$\omega_{0}=$ fundamental frequency of mono-dimensional approximation

$\tilde{\omega}_{i}=$ commensurate approximation of $\omega_{i}$

$(\sim)=$ for vectors: harmonic coefficients (frequency domain) entity

$\left(^{-}\right)=$multidimensional representation

$()=$, dot product

\section{References}

[1] L. Chua, and Ushida, A., 1981, "Algorithms for Computing Almost Periodic Steady State Response of Non-Linear Systems to Multiple Input Frequencies," IEEE Trans. Circuits Syst., 28(10), pp. 953-971.

[2] Schilder, F., Vogt, W., Schreiber, S., and Osinga, H., 2006, "Fourier Methods for Quasi-Periodic Oscillations,. Int. J. Numer. Methods Eng., 67(5), pp. 629-671.

[3] Hibner, D., 1975, "Dynamic Response of Viscous-Damped Multi-Shaft Jet Engines," J. Aircr., 12(4), pp. 305-312.

[4] M. Lalanne, and Ferraris, G., 1997, Rotordynamics Prediction in Engineering, 2nd ed., Wiley, New York.

[5] Ehrich, F., ed., 1998, Handbook of Rotordynamics. Krieger, Malabar, FL.

[6] Guskov, M., Sinou, J.-J., and Thouverez, F., 2007, "Multi-Dimensional Harmonic Balance Applied to Rotor Dynamics," Proceedings of the ASME International Design Engineering Technical Conferences and Computers and Information in Engineering Conference, DETC2007.

[7] Nayfeh, A., and Mook, D., 1979, Non-Linear Oscillations, John Wiley \& Sons, Inc. New York.

[8] Hayashi, C., 1953, Nonlinear Oscillations in Physical Systems, Princeton University, Princeton, NJ.

[9] Genesio, R., and Tesi, A., 1992, "Harmonic Balance Methods for the Analysis of Chaotic Dynamics in Nonlinear Systems," Automatica, 28(3), pp. 531-548.

[10] Hahn, E., and Chen, P., 1994, "Harmonic Balance Analysis of General Squeeze-Film Damped Multidegree-of-Freedom Rotor Bearing Systems," ASME J. Tribol., 116, pp. 499-507.

[11] Nacivet, S., Pierre, C., Thouverez, F., and Jezequel, L., 2003, "A Dynamic Lagrangian Frequency-Time Method for the Vibration of Dry-Friction-Damped Systems," J. Sound Vib., 265(1), pp. 201-219.

[12] Nandakumar, K., and Chatterjee, A., 2005, "Higher-Order Pseudo Averaging Via Harmonic Balance for Strongly Nonlinear Oscillations," ASME J. Vibr. Acoust., 127(4), pp. 416-419.

[13] Basso, M., Materassi, D., and Salapaka, M., 2008, "Hysteresis Models of Dynamic Mode Atomic Force Microscopes: Analysis and Identification Via Harmonic Balance,” Nonlinear Dyn., 54(4), pp. 297-306.

[14] Sundararajan, P., and Noah, S., 1997, "Dynamics of Forced Nonlinear Systems Using Shooting/Arc Length Continuation Method - Application To Rotor Systems," ASME J. Vibr. Acoust., 119, pp. 9-20.

[15] Rodrigues, F., Thouverez, F., Gibert, C., and Jezequel, L., 2003, "Chebyshev Polynomials Fits for Efficient Analysis of Finite Length Squeeze Film Damped Rotors," ASME J. Eng. Gas Turbines Power, 125(1), pp. 175-183.

[16] Dunne, J., and Hayward, P., 2006, "A Split-Frequency Harmonic Balance Method for Nonlinear Oscillators With Multiharmonic Forcing," J. Sound Vib., 295(3-5), pp. 939-963.

[17] Lau, S., and Cheung, Y., 1983, "Incremental Harmonic Balance Method With Multiple Time Scales for Aperiodic Vibration Of Nonlinear Systems," J. Appl. Mech., 50, pp. 871-876.

[18] Kim, Y.-B., and Noah, S., 1996, "Quasi-Periodic Response and Stability Analysis of a Non-Linear Jeffcott Rotor," J. Sound Vib., 190(2), pp. 239-253.

[19] Kim, Y., and Choi, S.-K., 1997, "A Multiple Harmonic Balance Method for the Internal Resonant Vibration of a Non-Linear Jeffcott Rotor," J. Sound Vib., 208(5), pp. 745-761.

[20] Pusenjak, R., and Oblak, M., 2004, "Incremental Harmonic Balance Method With Multiple Time Variables for Dynamical Systems With Cubic Non-Linearities," Int. J. Numer. Methods Eng., 59, pp. 255-292. 
[21] Akgün, D., Cankaya, I., and Peyton Jones, J., 2009, “A Symbolic Algorithm for the Automatic Computation of Multitone-Input Harmonic Balance Equations for Nonlinear Systems," Nonlinear Dyn., 56(1), pp. 179-191.

[22] Guskov, M., Sinou, J.-J., and Thouverez, F., 2008, "Multi-Dimensional Harmonic Balance Applied to Rotor Dynamics," Mech.Res. Commun., 35, pp. $537-545$.

[23] Legrand, M., 2005, "Modèles de Prédiction de l'Intéraction Rotor/Stator dans un Moteur d'Avion," $\mathrm{PhD}$ thesis, Université de Nantes, Nantes, France.

[24] Laxalde and, D., Thouverez, F., 2007, "Non-Linear Vibrations of Multi-Stage Bladed Disks Systems with Friction Ring Dampers," Proceedings of the ASME International Design Engineering Technical Conferences and Computers and Information in Engineering Conference, DETC2007.
[25] Kaas-Petersen, C., 1985, "Computation of Quasi-Periodic Solutions of Forced Dissipative Systems," J. Comput. Phys., 58, pp. 395-408.

[26] Kaas-Petersen, C., 1986, "Computation of Quasi-Periodic Solutions of Forced Dissipative Systems II," J. Comput. Phys., 64, pp. 433-442.

[27] Kaas-Petersen, C., 1987, "Computation, Continuation and Bifurcation of Torus Solutions for Dissipative Maps and Ordinary Differential Equations," Physica D, 25, pp. 288-306.

[28] Kim, Y., 1996, "Quasi-Periodic Response And Stability Analysis for NonLinear Systems: A General Approach," J. Sound Vib., 192(4), pp. 821-833.

[29] Tiwari, M., Gupta, K., and Prakash, O., 2000, "Effect of Radial Internal Clearance of a Ball Bearing on the Dynamics of a Balanced Horizontal Rotor," J. Sound Vib., 238(5), pp. 723-756. 